RECENT DEVELOPMENTS

\author{
IN FUNCTIONAL EQUATIONS AND INEQUALITIES \\ BANACH CENTER PUBLICATIONS, VOLUME 99 \\ INSTITUTE OF MATHEMATICS \\ POLISH ACADEMY OF SCIENCES \\ WARSZAWA 2013
}

\title{
ON CONJUGACY EQUATION IN DIMENSION ONE
}

\author{
KRZYSZTOF CIEPLIŃSKI and ZBIGNIEW LEŚNIAK \\ Department of Mathematics, Pedagogical University \\ Podchorązych 2, 30-084 Kraków, Poland \\ E-mail:kc@up.krakow.pl,zlesniak@up.krakow.pl
}

Dedicated to the memory of Professor Bogdan Choczewski

\begin{abstract}
In this paper, recent results on the existence and uniqueness of (continuous and homeomorphic) solutions $\varphi$ of the equation $\varphi \circ f=g \circ \varphi$ ( $f$ and $g$ are given self-maps of an interval or the circle) are surveyed. Some applications of these results as well as the outcomes concerning systems of such equations are also presented.
\end{abstract}

1. Introduction. In the paper we survey recent (i.e., published since 2000) results on conjugacy equation in dimension one. More precisely, we focus on the existence and uniqueness of (continuous and homeomorphic) solutions $\varphi$ of the functional equation

$$
\varphi \circ f=g \circ \varphi
$$

where $f$ and $g$ are given self-maps of a real interval or the unit circle $\mathbb{S}^{1}$. Moreover, we give applications of the presented results to investigating iterative roots. The outcomes on systems of such equations are also shown.

Let us recall that a homeomorphism $\varphi$ satisfying equation (1) is said to be a topological conjugacy between $f$ and $g$ ( $f$ and $g$ are then called topologically conjugate). If the function $\varphi$ fulfilling equation (1) is only a continuous surjection, then we say that it is a topological semi-conjugacy between $f$ and $g$, which are then called topologically semi-conjugate. These notions as well as their applications (for example, to dynamical systems and functional equations) are well-known; they can be found for instance in [1, 2, 11, 13, 14.

2010 Mathematics Subject Classification: Primary 39B12; Secondary 37C15, 37E05, 37E10, 39B22, 39B72.

Key words and phrases: conjugacy equation, topological semi-conjugacy, topological conjugacy. The paper is in final form and no version of it will be published elsewhere. 


\section{Self-maps of intervals}

2.1. The equation. Consider the class $\mathcal{M}$ consisting of all functions $f:[0,1] \rightarrow[0,1]$ for which there exists a partition $0=t_{0}<t_{1}<\ldots<t_{n}=1(n \geq 2)$ such that:

(i) for each $j \in\{1, \ldots, n\}$ the restriction $\left.f\right|_{\left(t_{j-1}, t_{j}\right)}$ is $C^{1}$ and strictly monotone,

(ii) for each $j \in\{1, \ldots, n\}$ there exist $p(j), q(j) \in\{0, \ldots, n\}$ with $p(j)<q(j)$ such that $f\left(\left(t_{j-1}, t_{j}\right)\right)=\left(t_{p(j)}, t_{q(j)}\right)$,

(iii) there exists a $\lambda>1$ such that $\left|f^{\prime}(x)\right| \geq \lambda$ for all $x \in(0,1) \backslash\left\{t_{1}, \ldots, t_{n-1}\right\}$.

In 2001, N. A. Fotiades and M. A. Boudourides proved the following result.

Theorem 2.1 (Theorem 2.7 in [8]). Let $f \in \mathcal{M}, 0=t_{0}<t_{1}<\ldots<t_{n}=1$ be the partition corresponding to $f$, and $f$ be continuous from the right or from the left at $t_{j}$ for $j \in\{0, \ldots, n\}$. Assume also that $T \in \mathcal{M}$ is the map with partition $0=s_{0}<s_{1}<$ $\ldots<s_{n}=1$, where $s_{j}=\frac{j}{n}$ for $j \in\{0, \ldots, n\}$, which is affine in each interval $\left(\frac{j-1}{n}, \frac{j}{n}\right)$ and $T\left(\left(\frac{j-1}{n}, \frac{j}{n}\right)\right)=\left(\frac{p(j)}{n}, \frac{q(j)}{n}\right)$ for $j \in\{1, \ldots, n\}$. Furthermore, let for each $j \in\{1, \ldots, n\}$ the restriction $\left.T\right|_{\left(s_{j-1}, s_{j}\right)}$ be of the same type of monotonicity as $\left.f\right|_{\left(t_{j-1}, t_{j}\right)}$, and for each $j \in\{0, \ldots, n\}, T$ be continuous from the right (from the left) at $s_{j}$, if $f$ is continuous from the right (from the left) at $t_{j}$. Then $T$ and $f$ are topologically conjugate.

Let $a, b \in \mathbb{R}$ be such that $a<b$, let $r$ be a positive integer and put $I:=[a, b]$. Recall that a continuous map $f: I \rightarrow I$ is said to be $r$-modal if there exists a partition $a=t_{0}<t_{1}<\ldots<t_{r+1}=b$ such that for each $j \in\{1, \ldots, r+1\}$ the restriction $\left.f\right|_{\left(t_{j-1}, t_{j}\right)}$ is strictly monotone and $\left(t_{j-1}, t_{j}\right)$ is the maximal open interval with this property. The intervals $\left(t_{j-1}, t_{j}\right)$ are then called monotone branches. If $r=1$, then we say that $f$ is a unimodal map.

An $r$-modal map $f: I \rightarrow I$ is called piecewise expanding if there exist a metric $d: I \times I \rightarrow \mathbb{R}$ (topologically equivalent to the Euclidean one) and a constant $\mu>1$ such that

$$
d(f(x), f(y)) \geq \mu d(x, y), \quad x, y \in\left(t_{j-1}, t_{j}\right), j \in\{1, \ldots, r+1\} .
$$

Two piecewise expanding $r$-modal maps $f, g: I \rightarrow I$ with partitions $\left\{t_{j}\right\}_{j=0}^{r+1}$ and $\left\{s_{j}\right\}_{j=0}^{r+1}$, respectively, are said to be combinatorially equivalent if there exists a homeomorphism $h: I \rightarrow I$ such that

$$
h\left(f^{m}\left(t_{j}\right)\right)=g^{m}\left(s_{j}\right), \quad j \in\{0, \ldots, r+1\}, m \in \mathbb{N} .
$$

Using the Banach fixed point theorem, C. Kawan proved the following result.

Theorem 2.2 (Theorem 3.7 in [9]). If $f, g: I \rightarrow I$ are combinatorially equivalent piecewise expanding $r$-modal maps, then they are topologically conjugate.

In the same paper, he also gave the following sufficient condition for piecewise expansiveness of $r$-modal maps.

Theorem 2.3 (Theorem 3.6 in [9]). Let $f: I \rightarrow I$ be an $r$-modal map with a partition $\left\{t_{i}\right\}_{i=0}^{r+1}$. Denote by $P$ the set of $t_{i}$ such that $t_{i} \notin f\left(\left(t_{j-1}, t_{j}\right)\right)$ for every $j \in\{1, \ldots, r+1\}$. Assume that $f$ is $C^{1}$ on $I \backslash P$. Let $\mu>1$ and $\Phi: I \rightarrow \mathbb{R}$ be a non-negative continuous 
function such that $\Phi^{-1}(\{0)\} \subset P$. If $\frac{1}{\Phi} \in L^{1}(I)$ and

$$
\left|f^{\prime}(x)\right| \geq \mu \frac{\Phi(f(x))}{\Phi(x)}, \quad x \in I \backslash P,
$$

then $f$ is piecewise expanding, where the metric $d$ occurring in 2 is given by

$$
d(x, y)=\left|\int_{x}^{y} \frac{d z}{\Phi(z)}\right|, \quad x, y \in I .
$$

Next, Kawan proved that the unimodal maps $f_{\alpha}:[-1,1] \rightarrow[-1,1]$ given, for any $\alpha>1$, by

$$
f_{\alpha}(x)=1-2|x|^{\alpha}
$$

are piecewise expanding. To do this he used Theorem 2.3 for $\Phi_{\alpha}(x)=\left(1-x^{2}\right)^{(\alpha-1) / \alpha}$ and $\mu_{\alpha}=\alpha^{1 / \alpha}$. Therefore, by Theorem 2.2 , the maps $f_{\alpha}$ are pairwise topologically conjugate.

Moreover, he applied the Banach fixed point theorem once more to show that for any $\alpha>4$ the logistic map $g_{\alpha}: \mathbb{R} \rightarrow \mathbb{R}$, given by

$$
g_{\alpha}(x)=\alpha x(1-x),
$$

is topologically conjugate to the tent map $g: \mathbb{R} \rightarrow \mathbb{R}$ of the form

$$
g(x)=\frac{3}{2}(1-|2 x-1|) .
$$

Let us point out that the maps $g_{\alpha}$ are not piecewise expanding, so Theorem 2.2 cannot be used in this case.

Let us next recall (see [4]) that a continuous function $f: I \rightarrow I$ is said to be a horseshoe map if there exists a partition $a=t_{0}<t_{1}<\ldots<t_{n}=b(n \geq 2)$ such that for each $j \in\{1, \ldots, n\}, f_{\mid\left[t_{j-1}, t_{j}\right]}$ is a homeomorphism of the interval $\left[t_{j-1}, t_{j}\right]$ (which is called a lap of $f$ ) onto $I$. If for each $j \in\{1, \ldots, n\}, f_{\mid\left[t_{j-1}, t_{j}\right]}:\left[t_{j-1}, t_{j}\right] \rightarrow I$ is a monotone surjection, then $f$ is called a weak horseshoe map. Moreover, we say that two horseshoe maps $f: I \rightarrow I$ and $g: J \rightarrow J$ (here and subsequently, $J:=[c, d]$ with some $c, d \in \mathbb{R}$ such that $c<d$ ) having the same number of laps are of the same type if $f$ and $g$ are of the same type of monotonicity on their leftmost laps.

Let $(X, d)$ be a metric space. A function $T: X \rightarrow X$ is called weakly contractive if

$$
d(T(x), T(y))<d(x, y), \quad x, y \in X, x \neq y .
$$

Furthermore, given an increasing function $\gamma:[0, \infty) \rightarrow[0, \infty)$ such that $\lim _{n \rightarrow \infty} \gamma^{n}(t)=0$ for $t \in(0, \infty)$, we say that $T: X \rightarrow X$ is $\gamma$-contractive if

$$
d(T(x), T(y)) \leq \gamma(d(x, y)), \quad x, y \in X .
$$

Given two horseshoe maps $f: I \rightarrow I$ and $g: J \rightarrow J$ having laps

$$
\left[t_{j-1}, t_{j}\right], \quad\left[s_{j-1}, s_{j}\right], \quad j \in\{1, \ldots, n\},
$$

respectively, for each $j \in\{0, \ldots, n-1\}$ put

$$
I_{j}:=\left[t_{j}, t_{j+1}\right], \quad J_{j}:=\left[s_{j}, s_{j+1}\right] \quad \text { and } \quad f_{j}:=f_{\mid I_{j}}, \quad g_{j}:=g_{\mid J_{j}} .
$$

The horseshoe map $f$ is called piecewise weakly expanding (respectively, piecewise $\gamma$-expanding) if for each $j \in\{0, \ldots, n-1\}, f_{j}^{-1}$ is weakly contractive (respectively, $\gamma$-contractive for a $\gamma:[0, \infty) \rightarrow[0, \infty))$. 
Proposition 2.4 (Proposition 2.2 in [6]). Assume that

(H) $f: I \rightarrow I$ and $g: J \rightarrow J$ are horseshoe maps of the same type and having $n$ laps $\left[t_{j-1}, t_{j}\right]$ and $\left[s_{j-1}, s_{j}\right]$, respectively,

and $g$ is piecewise weakly expanding. If $\varphi: I \rightarrow J$ is a continuous and non-constant solution of equation (1), then $\varphi$ is a topological semi-conjugacy. If, moreover, $n$ is even and $\varphi$ is injective, then $\varphi$ is an increasing topological conjugacy.

In the proofs of the following two theorems a fixed point principle from [12] plays a crucial role.

THEOREM 2.5 (Theorem 3.1 in [6]). If assumption $(\mathrm{H})$ holds and $g$ is piecewise $\gamma$-expanding, then there exists a unique function $\varphi: I \rightarrow J$ satisfying equation (1) and the condition

$$
\varphi\left[I_{j}\right] \subset J_{j}, \quad j \in\{0, \ldots, n-1\} .
$$

This function is an increasing topological semi-conjugacy. If, moreover, $f$ is piecewise weakly expanding, then $\varphi$ is also a topological conjugacy.

TheOrem 2.6 (Theorem 3.2 in [6]). If $n$ is odd, assumption $(\mathrm{H})$ holds and $g$ is piecewise $\gamma$-expanding, then there exists a unique function $\varphi: I \rightarrow J$ satisfying equation (1) and the condition

$$
\varphi\left[I_{i}\right] \subset J_{n-i-1}, \quad i \in\{0, \ldots, n-1\} .
$$

This function is a decreasing topological semi-conjugacy. If, moreover, $f$ is piecewise weakly expanding, then $\varphi$ is also a topological conjugacy.

Corollary 2.7 (Corollary 3.3 in [6]). If assumption $(\mathrm{H})$ holds, $n$ is odd and $g$ is piecewise $\gamma$-expanding, then equation (1) has exactly two monotone and surjective solutions. One of them is increasing, while the other is decreasing.

The next theorem gives some conditions guaranteeing the existence of topological conjugacies.

THEOREM 2.8 (Theorem 3.5 in [6]). If assumption $(\mathrm{H})$ holds, and $f$ and $g$ are piecewise weakly expanding, then there exists a unique function $\varphi: I \rightarrow J$ satisfying equation (1) and condition (3). This function is an increasing topological conjugacy. If, moreover, $n$ is odd, then there is also exactly one mapping $\varphi: I \rightarrow J$ fulfilling equation (1) and condition (4). This mapping is a decreasing topological conjugacy.

On the other hand, we have the following fact.

REMARK 2.9 (Remark 3.4 in [6]). If assumption (H) holds, $g$ is piecewise weakly expanding and $n$ is even, then there is no topological conjugacy satisfying condition (4).

Corollary 2.10 (Corollary 3.6 in [6]). Let assumption $(\mathrm{H})$ hold, and $f$ and $g$ be piecewise weakly expanding.

(i) If $n$ is even, then there is a unique topological conjugacy between $f$ and $g$. This conjugacy is increasing.

(ii) If $n$ is odd, then there are exactly two topological conjugacies between $f$ and $g$. One of them is increasing, while the other is decreasing. 
Let us next recall (see for instance [10]) that a continuous self-map $f$ of a metric space $X$ is said to be (topologically) transitive if for any non-empty open subsets $U, V$ of $X$ there is a positive integer $n$ such that $f^{n}(U) \cap V \neq \emptyset$.

It is known (see [4, 14]) that two horseshoe maps of the same type and without homtervals (i.e., intervals on which all their iterates are monotone) are topologically conjugate. So are also transitive horseshoe maps having two laps each, and, in this case, topological conjugacy is only one (see [2]). Moreover, if $f$ is transitive and $g:[0,1] \rightarrow[0,1]$ is an arbitrary function, then every increasing topological semi-conjugacy $\varphi: I \rightarrow[0,1]$ is a topological conjugacy (see [1]).

The following example shows that if we omit the assumption of the transitivity of $f$, then an increasing topological semi-conjugacy needs not to be injective even if $g$ is continuous and transitive and $f$ is continuous and piecewise monotone.

ExAmPle 2.11 (Example 3.7 in [6]). Let $n=2, a=c=0, b=d=1, t_{1}=s_{1}=\frac{1}{2}$,

$$
f(x)= \begin{cases}2 x, & x \in\left[0, \frac{1}{2}\right], \\ -x+\frac{3}{2}, & x \in\left[\frac{1}{2}, \frac{5}{6}\right], \\ -4 x+4, & x \in\left[\frac{5}{6}, 1\right],\end{cases}
$$

and $g$ be the standard tent map defined by

$$
g(x)=1-|2 x-1|, \quad x \in[0,1] .
$$

It is well-known (see for instance [10] which is a survey on transitive maps) that $g$ is transitive. Moreover, $g$ fulfils the assumption of Theorem 2.5 with $\gamma(x):=\frac{1}{2} x$ for $x \in[0, \infty)$ and

$$
|f(x)-f(y)| \geq|x-y|, \quad x, y \in I_{j}, j \in\{0,1\} .
$$

By Theorem 2.5 there exists a unique function $\varphi$ satisfying equation (1) and condition (3). This function is an increasing topological semi-conjugacy. However, $\varphi$ is not injective, and there is no topological conjugacy between $f$ and $g$.

Let us finally mention that assumption $(\mathrm{H})$ itself does not ensure the uniqueness of conjugacy (and topological semi-conjugacy), which follows from the example below.

ExAmPle 2.12 (Example 3.10 in [6]). If $J=I$ and $g=f$ is a horseshoe map, then for each positive integer $m$ the function $\varphi:=f^{m}$ is a topological semi-conjugacy between $f$ and $g$. This function satisfies neither (3) nor (4). Thus, in this case there are infinitely many topological semi-conjugacies between $f$ and $g$.

In the considerations of Y.-G. Shi (see [17]) concerning (continuous) solutions of equation (1), the sets of periodic points of $f$ and $g$ play an important role. In order to present Shi's results, assume that $f$ and $g$ are continuous self-maps of the interval $I$ and for all $k \in \mathbb{N}$ consider the subsets $P_{k}(f)=\left\{x \in I: f^{k}(x)=x\right\}$ and $P_{k}(g)=\left\{x \in I: g^{k}(x)=x\right\}$ of the sets of all periodic points Per $f$ and Per $g$ of $f$ and $g$, respectively.

Let us start with the following lemma. 
LEMMA 2.13 (Lemma 2 in [17]). Let $f$ and $g$ be continuous self-maps of the interval $I$ and $k \in \mathbb{N}$. Then the general solution $\varphi$ of equation (1) satisfies

$$
\varphi(x)=\alpha_{1}(x), \quad x \in P_{1}(f),
$$

and

$$
\varphi(x)=\alpha_{2}(x), \quad x \in P_{k}(f) \backslash P_{1}(f),
$$

where $\alpha_{1}$ is a function from $P_{1}(f)$ into $P_{1}(g)$ if neither $P_{1}(f)$ nor $P_{1}(g)$ is empty and $\alpha_{2}$ is a function from $P_{k}(f) \backslash P_{1}(f)$ into $P_{j}(g)$ for some positive integer $j$ such that $j$ divides $k$, if neither $P_{k}(f) \backslash P_{1}(f)$ nor $P_{j}(g)$ is empty.

Now, we present the result of Y.-G. Shi in the case where $f$ is strictly decreasing.

Theorem 2.14 (Theorem 2 in [17]). Let $f$ be a strictly decreasing continuous function from $I$ onto itself and $g: I \rightarrow I$ be a continuous function. Assume that the set Per $f=P_{2}(f)$ is countable. Then equation (1) has infinitely many solutions continuous on $I \backslash$ Per $f$. Each of these solutions can be constructed in the following way: for each consecutive periodic points $p$ and $q$ of $f$ (i.e., $f$ has no periodic point on the interval $(p, q)$ ) choose initial points $x_{0} \in(p, q)$ and $y_{0} \in I$ and a continuous function $\varphi_{0}$ on $\left[x_{0}, f^{2}\left(x_{0}\right)\right]\left(\right.$ or $\left.\left[f^{2}\left(x_{0}\right), x_{0}\right]\right)$ such that $\varphi_{0}\left(x_{0}\right)=y_{0}$ and $\varphi_{0}\left(f^{2}\left(x_{0}\right)\right)=g^{2}\left(y_{0}\right)$ and define $\varphi$ on $(p, q) \cup(f(q), f(p))$ by

$$
\varphi(x)= \begin{cases}\varphi_{0}(x), & x \in\left[x_{0}, f^{2}\left(x_{0}\right)\right)\left(\text { or } x \in\left[f^{2}\left(x_{0}\right), x_{0}\right]\right), \\ g^{n}\left(\varphi_{0}\left(f^{-n}(x)\right)\right), & x \in\left[f^{n}\left(x_{0}\right), f^{n+2}\left(x_{0}\right)\right)\left(\text { or } x \in\left[f^{n+2}\left(x_{0}\right), f^{n}\left(x_{0}\right)\right]\right),\end{cases}
$$

where $n \in \mathbb{Z}$. The values of $\varphi$ on periodic points of $f$ are determined by Lemma 2.13 .

REMARK 2.15. The solution $\varphi$ of equation (1) described in Theorem 2.14 can be discontinuous at points of the set $\operatorname{Per} f$.

For any integer $n \geq 2$ denote by $T_{n}, T_{n^{-}}:[0,1] \rightarrow[0,1]$ the piecewise monotone and affine mappings defined as follows:

$$
\begin{gathered}
T_{n}\left(\frac{k}{n}\right)= \begin{cases}0, & k \in[0, n] \text { is an even integer, } \\
1, & k \in[0, n] \text { is an odd integer, }\end{cases} \\
T_{n^{-}}\left(\frac{k}{n}\right)= \begin{cases}1, & k \in[0, n] \text { is an even integer, } \\
0, & k \in[0, n] \text { is an odd integer, }\end{cases}
\end{gathered}
$$

and $T_{n}, T_{n^{-}}$are affine between these points. Clearly, $T_{2}$ is the standard tent map.

With the above notation we have the following theorem due to D.-S. Ou and K. J. Palmer.

TheOREM 2.16 (Theorem 1.1 in [16]). If $f:[0,1] \rightarrow[0,1]$ is a weak horseshoe map having $n$ laps and $f(0)=0$, then there exists a unique increasing topological semi-conjugacy $\varphi:[0,1] \rightarrow[0,1]$ between $f$ and $T_{n}$.

Let us next recall (see for instance [3]) that we say that a continuous map $f:[0,1] \rightarrow$ $[0,1]$ has an $n$-horseshoe if there exist $n$ closed subintervals of $[0,1], A_{1}, \ldots, A_{n}$, with pairwise disjoint interiors, such that

$$
\left(A_{1} \cup \ldots \cup A_{n}\right) \subset\left(f\left(A_{1}\right) \cap \ldots \cap f\left(A_{n}\right)\right) .
$$


A continuous function $f:[0,1] \rightarrow[0,1]$ is called turbulent if it has a 2-horseshoe.

Very recently, L. Block, J. Keesling and D. Ledis proved the following result.

THEOREM 2.17 (Theorem 3.12 in [3]). If $f:[0,1] \rightarrow[0,1]$ is a turbulent map, then $f$ and the standard tent map $T_{2}$ are topologically semi-conjugate.

REMARK 2.18. The topological semi-conjugacy between a turbulent map and the standard tent map is not necessarily unique. In the proof of Remark 3.18 in [3] two different topological semi-conjugacies between $T_{4}$ and $T_{2}$ were provided. Let us also mention here (see Proposition 3.19 in [3]) that these semi-conjugacies are not monotone.

The example below shows that the converse of Theorem 2.17 is not true.

EXAMPLE 2.19 (see the proof of Theorem 3.13 in [3]). Let $f:[0,1] \rightarrow[0,1]$ be given by

$$
f(x)= \begin{cases}2 x+\frac{1}{2}, & x \in\left[0, \frac{1}{4}\right], \\ -2 x+\frac{3}{2}, & x \in\left[\frac{1}{4}, \frac{3}{4}\right], \\ 2 x-\frac{3}{2}, & x \in\left[\frac{3}{4}, 1\right] .\end{cases}
$$

Then $f$ is not turbulent, while $f$ and $T_{2}$ are topologically semi-conjugate.

The main result of [3] is the following theorem.

THEOREM 2.20 (Theorem 3.31 in [3]). Let $f:[0,1] \rightarrow[0,1]$ be a continuous mapping having an $n$-horseshoe for an integer $n \geq 2$. Then there exists a topological semi-conjugacy between $f$ and $T_{n}$ or $T_{n^{-}}$.

Denote by $\mathcal{M}_{r}(I)$ the set of all $r$-modal maps $f: I \rightarrow I$ and put

$$
\begin{array}{r}
\mathcal{M}_{r}^{1}(I):=\left\{f \in \mathcal{M}_{r}(I): f(x)<x \text { for } x \in\left(t_{0}, t_{1}\right], f\left(t_{2 j}\right)=t_{0} \text { for } j \in\left\{0, \ldots,\left\lfloor\frac{r}{2}\right\rfloor\right\},\right. \\
\left.f\left(t_{2 j-1}\right)=f\left(t_{1}\right) \geq f\left(t_{r+1}\right) \text { for } j \in\left\{2, \ldots,\left\lfloor\frac{r+1}{2}\right\rfloor\right\}\right\},
\end{array}
$$

where $\left\{t_{j}\right\}_{j=0}^{r+1}$ is the partition of $f$.

With this notation we have the following results (here and subsequently $\left\{s_{j}\right\}_{j=0}^{r+1}$ stands for the partition of $g$ ).

THEOREM 2.21 (Theorem 1 in [18]). Let $f \in \mathcal{M}_{r}^{1}(I)$ and $g \in \mathcal{M}_{r}^{1}(J)$. Then $f$ and $g$ are topologically conjugate if and only if there exists a positive integer $m$ such that one of the following conditions holds:

(i) $f\left(t_{r+1}\right)=f^{m}\left(t_{1}\right), g\left(s_{r+1}\right)=g^{m}\left(s_{1}\right)$;

(ii) $f\left(t_{r+1}\right)=t_{0}, g\left(s_{r+1}\right)=s_{0}$;

(iii) $f\left(t_{r+1}\right) \in\left(f^{m+1}\left(t_{1}\right), f^{m}\left(t_{1}\right)\right), g\left(s_{r+1}\right) \in\left(g^{m+1}\left(s_{1}\right), g^{m}\left(s_{1}\right)\right)$.

Furthermore, for cases (i) and (ii), any homeomorphism $\varphi_{0}:\left[f\left(t_{1}\right), t_{1}\right] \rightarrow\left[g\left(s_{1}\right), s_{1}\right]$ such that

$$
\varphi_{0}\left(t_{1}\right)=s_{1}, \quad \varphi_{0}\left(f\left(t_{1}\right)\right)=g\left(s_{1}\right)
$$

can be uniquely extended to a homeomorphic solution of equation (1) on I, as well as for case (iii) with the additional condition

$$
\varphi_{0}\left(f_{\mid\left[t_{0}, t_{1}\right]}^{-m}\left(f\left(t_{r+1}\right)\right)\right)=g_{\mid\left[s_{0}, s_{1}\right]}^{-m}\left(g\left(s_{r+1}\right)\right) .
$$


THEOREM 2.22 (Theorem 2 in [18). Under the assumptions of Theorem 2.21, any homeomorphism $\varphi_{0}:\left[f\left(t_{1}\right), t_{1}\right] \rightarrow\left[g\left(s_{1}\right), s_{1}\right]$ satisfying (5) (and (6) for case (iii)) can be extended to finitely many continuous non-monotone solutions of equation (1). In case $I=J$ only one of these solutions belongs to $\mathcal{M}_{r}^{1}(I)$.

Now, we give an application of the presented results to investigating iterative roots. Before we do this, let us recall that for a given integer $k \geq 2$, a self-map $f$ of a set $X$ is said to be an iterative root (of order $k$ ) of a function $g: X \rightarrow X$ if $f^{k}=g$.

Using Theorem 2.21, one can obtain the form of all iterative roots $f \in \mathcal{M}_{r}^{1}(I)$ of some $g \in \mathcal{M}_{r}^{1}(I)$ :

Theorem 2.23 (Corollary 2 in [18]). Let $k \geq 2$ be an integer and $g \in \mathcal{M}_{r}^{1}(I)$ be such that $g\left(s_{r+1}\right) \in\left\{s_{0}, g\left(s_{1}\right)\right\}$. Then every iterative root $f \in \mathcal{M}_{r}^{1}(I)$ of order $k$ of the function $g$ is given by

$$
f=\varphi^{-1} \circ g \circ \varphi,
$$

where $\varphi$ is a topological conjugacy between $g$ and $g^{k}$.

2.2. The system. Let $(X, \rho)$ be a complete metric space and $n \geq 2$ be an integer. In [20], M. C. Zdun investigated bounded and continuous solutions $\varphi:[0,1] \rightarrow X$ of the system of functional equations

$$
\varphi\left(f_{k}(x)\right)=F_{k}(\varphi(x)), \quad k \in\{0, \ldots, n-1\},
$$

where given functions $f_{0}, \ldots, f_{n-1}:[0,1] \rightarrow[0,1]$ and $F_{0}, \ldots, F_{n-1}: X \rightarrow X$ satisfy at least one of the following hypotheses:

(H1) $f_{0}, \ldots, f_{n-1}$ are continuous, strictly increasing, $f_{0}(0)=0, f_{n-1}(1)=1$ and $f_{k+1}(0)$ $=f_{k}(1)$ for $k \in\{0, \ldots, n-2\}$

(H2) $F_{0}, \ldots, F_{n-1}$ are continuous, $F_{0}$ and $F_{n-1}$ have unique fixed points $a$ and $b$, respectively, and $F_{k+1}(a)=F_{k}(b)$ for $k \in\{0, \ldots, n-2\}$.

The assumption that $a$ and $b$ are the unique fixed points of $F_{0}$ and $F_{n-1}$ implies that for any solution $\varphi:[0,1] \rightarrow X$ of system (7) we have $\varphi(0)=a$ and $\varphi(1)=b$ (see Remark 1 in [20]).

TheOREM 2.24 (see Theorem 1 in [20]). Assume that the mappings $f_{0}, \ldots, f_{n-1}$ fulfil (H1), and there exists an increasing function $\alpha:[0, \infty) \rightarrow[0, \infty)$ such that the sequence of its iterates converges pointwise to the zero function on $[0, \infty)$ and

$$
\rho\left(F_{k}(x), F_{k}(y)\right) \leq \alpha(\rho(x, y)), \quad x, y \in X, k \in\{0, \ldots, n-1\},
$$

(i.e., each $F_{k}$ is $\alpha$-contractive). Then for any $c_{1}, \ldots, c_{n-1} \in X$ there exists a unique bounded solution $\varphi:(0,1) \rightarrow X$ of (7) such that $\varphi\left(f_{k}(0)\right)=c_{k}$ for $k \in\{1, \ldots, n-1\}$. Moreover, if $F_{0}, \ldots, F_{n-1}$ satisfy $(\mathrm{H} 2)$, then there exists a unique bounded solution $\varphi:[0,1] \rightarrow X$ of system (7). This solution is continuous.

Under the assumption that the mappings $F_{0}, \ldots, F_{n-1}$ satisfy (H2), every bounded solution $\varphi:(0,1) \rightarrow X$ of system (7) can be extended to $[0,1]$ if $c_{k}=F_{k}(a)$ for $k \in\{1, \ldots, n-1\}$. Moreover, in the case where the functions $f_{0}, \ldots, f_{n-1}$ are given 
by

$$
f_{k}(x)=\frac{x+k}{n}, \quad x \in[0,1], k \in\{0, \ldots, n-1\},
$$

the theorem below provides a formula for a solution of 7 .

THEOREM 2.25 (Theorem 2 in [20]). Assume that the mappings $F_{0}, \ldots, F_{n-1}$ satisfy $(\mathrm{H} 2)$, and there exists an increasing function $\alpha:[0, \infty) \rightarrow[0, \infty)$ such that the sequence of its iterates converges pointwise to the zero function on $[0, \infty)$ and $(8)$ holds. Then the unique bounded solution $\varphi:[0,1] \rightarrow X$ of the system

$$
\varphi\left(\frac{x+k}{n}\right)=F_{k}(\varphi(x)), \quad k \in\{0, \ldots, n-1\},
$$

is given by

$$
\varphi(x)=\lim _{\nu \rightarrow \infty} F_{k_{1}} \circ \ldots \circ F_{k_{\nu}}(\xi), \quad x \in[0,1]
$$

where $\xi$ is an arbitrary element of $X$ and $k_{i} \in\{0, \ldots, n-1\}$ for $i \in \mathbb{N}$ are digits of the representation of $x$ in a positional base-n numeral system, i.e.,

$$
x=\sum_{i=1}^{\infty} \frac{k_{i}}{n^{i}} .
$$

The next theorem describes the connection between continuous solutions of system (7) and a (unique) fixed point of the Barnsley-Hutchinson operator associated with the Iterated Function System $F_{0}, \ldots, F_{n-1}$.

TheOrem 2.26 (Theorem 3 in [20]). Let the mappings $f_{0}, \ldots, f_{n-1}$ fulfil (H1). Assume also that $F_{0}, \ldots, F_{n-1}$ satisfy $(\mathrm{H} 2)$ and (8) with an increasing function $\alpha:[0, \infty) \rightarrow$ $[0, \infty)$ such that $\lim _{x \rightarrow t^{+}} \alpha(x)=\alpha(t)<t$ for $t \in(0, \infty)$. Then a non-empty compact set $C \subset X$ is a curve parameterized by a continuous solution $\varphi$ of system (7), i.e., $C=\{\varphi(t): t \in[0,1]\}$, if and only if $C$ is a unique fixed point of the Barnsley-Hutchinson operator

$$
F(A):=\bigcup_{i=0}^{n-1} F_{i}[A], \quad A \in c(X),
$$

where $c(X)$ denotes the metric space of non-empty compact subsets of $(X, \rho)$ with the Hausdorff metric $d_{\rho}$ generated by the metric $\rho$. Moreover, the curve $C$ does not depend on the choice of the system of functions $f_{0}, \ldots, f_{n-1}$ satisfying $(\mathrm{H} 1)$.

REMARK 2.27. The assumption of Theorem 2.26 on the function $\alpha$ implies that the Barnsley-Hutchinson operator satisfies the relation

$$
d_{\rho}(F(A), F(B)) \leq \alpha\left(d_{\rho}(A, B)\right), \quad A, B \in c(X) .
$$

In the case where $X=J$ is an interval (bounded or unbounded) which is a closed subset of $\mathbb{R}$, the following two results give more information on solutions of system (7).

TheOREM 2.28 (Theorem 4 in [20]). Assume that the mappings $f_{0}, \ldots, f_{n-1}$ satisfy (H1) and $F_{0}, \ldots, F_{n-1}: J \rightarrow J$ are increasing functions fulfilling $(\mathrm{H} 2)$ and (8) with an increasing function $\alpha:[0, \infty) \rightarrow[0, \infty)$ such that the sequence of its iterates converges pointwise to the zero function on $[0, \infty)$. Then the unique bounded solution $\varphi:[0,1] \rightarrow J$ of system (7) is monotone. 
TheOREM 2.29 (Theorem 5 in [20]). Let the mappings $f_{0}, \ldots, f_{n-1}$ satisfy $(\mathrm{H} 1)$ and

$$
\left|f_{k}(x)-f_{k}(y)\right|<|x-y|, \quad x \neq y, x, y \in[0,1], k \in\{0, \ldots, n-1\} .
$$

Assume also that $F_{0}, \ldots, F_{n-1}: J \rightarrow J$ are strictly increasing functions fulfilling (H2) with $a \neq b$ and

$$
\left|F_{k}(x)-F_{k}(y)\right|<|x-y|, \quad x \neq y, x, y \in J, k \in\{0, \ldots, n-1\} .
$$

Then the unique bounded solution $\varphi:[0,1] \rightarrow J$ of system (7) is strictly monotone. Moreover, if the interval $J$ is compact, then (7) has a unique solution.

\section{Self-maps of the circle}

3.1. Preliminaries. We begin this part of the paper by recalling the basic definitions and facts concerning self-maps of the unit circle.

It is well-known (see for instance [1]) that for every continuous mapping $F: \mathbb{S}^{1} \rightarrow \mathbb{S}^{1}$ there exist a continuous function $f: \mathbb{R} \rightarrow \mathbb{R}$, which is unique up to translation by an integer, and a unique integer $k$ such that

$$
F\left(e^{2 \pi i x}\right)=e^{2 \pi i f(x)}, \quad x \in \mathbb{R},
$$

and

$$
f(x+1)=f(x)+k, \quad x \in \mathbb{R} .
$$

The function $f$ is said to be the lift of $F$ and the integer $k$ is called the degree of $F$, and is denoted by $\operatorname{deg} F$. If $F$ is a homeomorphism, then so is its lift. Moreover, $|\operatorname{deg} F|=1$. We say that a homeomorphism $F: \mathbb{S}^{1} \rightarrow \mathbb{S}^{1}$ preserves (respectively, reverses) orientation if $\operatorname{deg} F=1$ (respectively, $\operatorname{deg} F=-1$ ), which is equivalent to the fact that the lift of $F$ is increasing (respectively, decreasing).

For an orientation-preserving homeomorphism $F: \mathbb{S}^{1} \rightarrow \mathbb{S}^{1}$ the number $\alpha(F) \in[0,1)$ defined by

$$
\alpha(F):=\lim _{n \rightarrow \infty} \frac{f^{n}(x)}{n}(\bmod 1), \quad x \in \mathbb{R},
$$

is called the rotation number of $F$. This number always exists and does not depend on $x$ and $f$. Furthermore, $\alpha(F) \in \mathbb{Q}$ if and only if $F$ has a periodic point. If $\alpha(F) \notin \mathbb{Q}$, then the non-empty set

$$
L_{F}:=\left\{F^{n}(z): n \in \mathbb{Z}\right\}^{d}, \quad z \in \mathbb{S}^{1},
$$

(the limit set of $F$ ) does not depend on $z$, is invariant with respect to $F$ and either $L_{F}=\mathbb{S}^{1}$ or $L_{F}$ is a perfect nowhere dense subset of $\mathbb{S}^{1}$ (see for instance [7]).

3.2. The equation. Given a continuous mapping $F: \mathbb{S}^{1} \rightarrow \mathbb{S}^{1}$, denote by $f$ its lift such that $f(0) \in[0,1)$. For any integer $n \geq 2$ put

$$
\mathcal{K}_{n}:=\left\{F: \mathbb{S}^{1} \rightarrow \mathbb{S}^{1}: F \text { is continuous, } \operatorname{deg} F=n\right.
$$

$$
\text { and }|x-y|<|f(x)-f(y)|, x, y \in \mathbb{R}, x \neq y\}
$$


and

$\mathcal{K}_{n}^{*}:=\left\{F: \mathbb{S}^{1} \rightarrow \mathbb{S}^{1}: F\right.$ is continuous, $\operatorname{deg} F=n$

and there is an increasing function $\gamma:[0, \infty) \longrightarrow[0, \infty)$ such that

$$
\left.\lim _{m \rightarrow \infty} \gamma^{m}(t)=0, t \in[0, \infty) \text { and }|x-y| \leq \gamma(|f(x)-f(y)|), x, y \in \mathbb{R}\right\} .
$$

With fixed $a, b \in \mathbb{S}^{1}$ and an integer $n \geq 2$, we have following three theorems due to M. C. Zdun (the last of them deals with the special case when $F(z)=z^{n}$ ).

THEOREM 3.1 (Theorem 1 in [19]). If $F, G \in \mathcal{K}_{n}$ and $F(a)=a, G(b)=b$, then for every integer $r \neq 0$ there exists a unique continuous solution $\Phi: \mathbb{S}^{1} \rightarrow \mathbb{S}^{1}$ of the equation

$$
\Phi(F(z))=G(\Phi(z)), \quad z \in \mathbb{S}^{1},
$$

such that $\operatorname{deg} \Phi=r, \Phi(a)=b$ and $\varphi[[0,1]]=[\varphi(0), \varphi(1)]$, where $\varphi$ is the lift of $\Phi$. Moreover, the mapping $\varphi$ is strictly monotone, and $\Phi$ is a topological conjugacy if and only if $|r|=1$.

THEOREM 3.2 (Theorem 2 in [19]). If $F \in \mathcal{K}_{n}, G \in \mathcal{K}_{n}^{*}$ and $F(a)=a, G(b)=b$, then for every integer $r \neq 0$ there exists a unique continuous solution $\Phi: \mathbb{S}^{1} \rightarrow \mathbb{S}^{1}$ of equation (9) such that $\operatorname{deg} \Phi=r$ and $\Phi(a)=b$. Moreover, the lift of $\Phi$ is strictly monotone.

THEOREM 3.3 (Theorem 4 in [19]). If $G \in \mathcal{K}_{n}$ and $G(a)=a$, then for every integer $r \neq 0$ there exists a unique continuous solution $\Phi: \mathbb{S}^{1} \rightarrow \mathbb{S}^{1}$ of the equation

$$
\Phi\left(z^{n}\right)=G(\Phi(z)), \quad z \in \mathbb{S}^{1},
$$

such that $\operatorname{deg} \Phi=r$ and $\Phi(1)=a$. If $|r|=1$, then $\Phi$ is a topological conjugacy.

Theorem 3.3 can be applied for proving the following result concerning continuous iterative roots of mappings from the class $\mathcal{K}_{n}$.

THEOREM 3.4 (Theorem 5 in [19]). Let $n \geq 2$ be an integer. A function $G \in \mathcal{K}_{n}$ has a continuous iterative root of order $k$ if and only if $n=r^{k}$ for an integer $r$.

With the notation

$$
\mathcal{S}_{r}:=\left\{\Psi: \mathbb{S}^{1} \rightarrow \mathbb{S}^{1}: \text { there is a homeomorphism } \Phi: \mathbb{S}^{1} \rightarrow \mathbb{S}^{1}\right.
$$

$$
\text { such that } \left.\Psi(z)=\Phi\left(\Phi^{-1}(z)^{r}\right), z \in \mathbb{S}^{1}\right\}
$$

we also have

THEOREM 3.5 (Theorem 6 in [19]). Let $k, n, r \geq 2$ be integers such that $n=r^{k}$. If $G \in \mathcal{K}_{n}$, then $G$ has exactly $\frac{n-1}{r-1}$ iterative roots of order $k$ in the class $\mathcal{S}_{r}$. They are given by the formula

$$
F(z)=\Phi\left(e^{2 \pi i(r-1) j /(n-1)} \Phi^{-1}(z)^{r}\right), \quad z \in \mathbb{S}^{1}, j=0, \ldots, \frac{n-r}{r-1},
$$

where $\Phi: \mathbb{S}^{1} \rightarrow \mathbb{S}^{1}$ is a homeomorphic solution of equation 10 .

In 2007 (see 21]), M. C. Zdun gave a necessary and sufficient condition for topological conjugacy of homeomorphisms of the circle having periodic points. Since stating this result (Theorem 17 in [21]) requires a long and sophisticated introduction, its details are omitted here. 
On the other hand, L. Block, J. Keesling and D. Ledis proved the following theorem. Theorem 3.6 (Theorem 6.7 in [3]). If $F, G: \mathbb{S}^{1} \rightarrow \mathbb{S}^{1}$ are continuous mappings with

$$
0<\operatorname{deg} F \neq \operatorname{deg} G>1 \text {, }
$$

then they are not topologically semi-conjugate.

It is well-known (see for instance [15]) that if two orientation-preserving homeomorphisms $F, G: \mathbb{S}^{1} \rightarrow \mathbb{S}^{1}$ are topologically conjugate, then either $\alpha(G)=\alpha(F)$ or $\alpha(G)+\alpha(F)=1$. The following theorem from [5] generalizes this fact.

TheOREM 3.7 (Theorem 1 in [5]). Let $F, G: \mathbb{S}^{1} \rightarrow \mathbb{S}^{1}$ be orientation-preserving homeomorphisms and suppose that there exists a continuous function $\Phi: \mathbb{S}^{1} \rightarrow \mathbb{S}^{1}$ such that (9) holds. Then

$$
\alpha(G)=\alpha(F) \operatorname{deg} \Phi(\bmod 1) .
$$

The proposition below is an extension of the well-known result of Poincaré.

Proposition 3.8 (Proposition 1 in [5]). If $F: \mathbb{S}^{1} \rightarrow \mathbb{S}^{1}$ is an orientation-preserving homeomorphism for which $\alpha(F) \notin \mathbb{Q}$, then there exists a unique continuous function $\Phi_{F}: \mathbb{S}^{1} \rightarrow \mathbb{S}^{1}$ such that

$$
\Phi_{F}(F(z))=e^{2 \pi i \alpha(F)} \Phi_{F}(z), \quad z \in \mathbb{S}^{1},
$$

and $\Phi_{F}(1)=1$. Moreover, $\operatorname{deg} \Phi_{F}=1$ and $\Phi_{F}$ is a homeomorphism if and only if $L_{F}=\mathbb{S}^{1}$.

Let $F: \mathbb{S}^{1} \rightarrow \mathbb{S}^{1}$ be an orientation-preserving homeomorphism such that $\alpha(F) \notin \mathbb{Q}$. The set

$$
K_{F}:=\Phi_{F}\left[\mathbb{S}^{1} \backslash L_{F}\right],
$$

where $\Phi_{F}: \mathbb{S}^{1} \rightarrow \mathbb{S}^{1}$ is the continuous solution of equation 11 with $\Phi_{F}(1)=1$, is said to be the iterative kernel of $F$.

The next two results provide some information about the properties of continuous (and homeomorphic) solutions of equation $(9)$ and justify some assumptions made in Theorem 3.12 (and Remark 3.13.

Theorem 3.9 (Theorem 2 in [5]). Let $F, G: \mathbb{S}^{1} \rightarrow \mathbb{S}^{1}$ be orientation-preserving homeomorphisms and suppose that $\alpha(G) \notin \mathbb{Q}$. If a continuous function $\Phi: \mathbb{S}^{1} \rightarrow \mathbb{S}^{1}$ of degree $l$ satisfies equation (9), then it is a topological semi-conjugacy and $\Phi\left[L_{F}\right]=L_{G}$. If, moreover, $L_{G} \neq \mathbb{S}^{1}$ and $d:=\Phi_{G}(\Phi(1))$, then for every $w \in \mathbb{S}^{1}$, dw $w^{l} \in K_{G}$ implies $w \in K_{F}$.

Proposition 3.10 (see Lemma 4 in [5]). Let $F, G: \mathbb{S}^{1} \rightarrow \mathbb{S}^{1}$ be orientation-preserving homeomorphisms and suppose that $\alpha(G) \notin \mathbb{Q}$. If $F$ and $G$ are topological conjugate via an orientation-preserving (respectively, orientation-reversing) homeomorphism $\Phi: \mathbb{S}^{1} \rightarrow \mathbb{S}^{1}$, then $K_{G}=d K_{F}$ (respectively, $\left.K_{G}=d K_{F}^{-1}\right)$ for $d:=\Phi_{G}(\Phi(1))$.

3.3. The system. In this part of the paper we present some results from [5], which concern continuous and homeomorphic solutions of the system

$$
\Phi\left(F_{t}(z)\right)=G_{t}(\Phi(z)), \quad z \in \mathbb{S}^{1}, t \in M,
$$


where $M$ is an arbitrary non-empty set and $F_{t}, G_{t}: \mathbb{S}^{1} \rightarrow \mathbb{S}^{1}$ for $t \in M$ are orientationpreserving homeomorphisms.

Theorem 3.11 (Theorem 3 in [5]). Let

$$
F_{t} \circ F_{s}=F_{s} \circ F_{t}, \quad G_{t} \circ G_{s}=G_{s} \circ G_{t}, \quad s, t \in M,
$$

and

$$
\alpha\left(G_{t}\right)=l \alpha\left(F_{t}\right)(\bmod 1), \quad t \in M,
$$

for an $l \in \mathbb{Z}$. Assume also that there is a $t_{0} \in M$ for which $\alpha\left(G_{t_{0}}\right) \notin \mathbb{Q}$.

(i) If $L_{G_{t_{0}}}=\mathbb{S}^{1}$, then for every $a \in \mathbb{S}^{1}$ there exists a unique continuous solution $\Phi: \mathbb{S}^{1} \rightarrow \mathbb{S}^{1}$ of system (12) such that $\Phi(1)=$ a. This solution is of degree l. If, moreover, $L_{F_{t_{0}}}=\mathbb{S}^{1}$ and $|l|=1$, then $\Phi$ is a homeomorphism.

(ii) If $L_{G_{t_{0}}} \neq \mathbb{S}^{1}$ and $L_{F_{t_{0}}}=\mathbb{S}^{1}$, then system (12) has no continuous solution.

THEOREM 3.12 (Theorem 4 in [5]). Let condition (14] hold for an $l \in \mathbb{Z}$ and there exist a $t_{0} \in M$ with $\alpha\left(G_{t_{0}}\right) \notin \mathbb{Q}$. Moreover, suppose that for all $k \in \mathbb{N}, t_{1}, \ldots, t_{k} \in M$ and $n_{1}, \ldots, n_{k} \in \mathbb{Z}$ such that $n_{1} \alpha\left(F_{t_{1}}\right)+\ldots+n_{k} \alpha\left(F_{t_{k}}\right) \in \mathbb{Z}$ we have

$$
F_{t_{1}}^{n_{1}} \circ \ldots \circ F_{t_{k}}^{n_{k}}=\mathrm{id}=G_{t_{1}}^{n_{1}} \circ \ldots \circ G_{t_{k}}^{n_{k}} .
$$

If $L_{F_{t_{0}}} \neq \mathbb{S}^{1} \neq L_{G_{t_{0}}}$ and there is a $d \in \mathbb{S}^{1}$ such that for every $w \in \mathbb{S}^{1}$, dw $w^{l} \in K_{G_{t_{0}}}$ implies $w \in K_{F_{t_{0}}}$, then system (12) has a continuous solution of degree $l$ depending on an arbitrary function.

REMARK 3.13 (Remark 3 in [5]). Under the hypotheses of Theorem 3.12 if, moreover, $|l|=1$ and $K_{G_{t_{0}}}=d K_{F_{t_{0}}}^{l}$ for a $d \in \mathbb{S}^{1}$, then system (12) has a homeomorphic solution of degree $l$ depending on an arbitrary function and the construction given in the proof of Theorem 3.12 (see [5]) determines all such solutions of (12).

\section{References}

[1] L. Alsedà, J. Llibre, M. Misiurewicz, Combinatorial Dynamics and Entropy in Dimension One, Adv. Ser. Nonlinear Dynam. 5, World Scientific, River Edge, NJ, 2000.

[2] J. Banks, V. Dragan, A. Jones, Chaos: a Mathematical Introduction, Austral. Math. Soc. Lect. Ser. 18, Cambridge Univ. Press, Cambridge, 2003.

[3] L. Block, J. Keesling, D. Ledis, Semi-conjugacies and inverse limit spaces, J. Difference Equ. Appl. 18 (2012), 627-645.

[4] A. Blokh, E. Coven, M. Misiurewicz, Z. Nitecki, Roots of continuous piecewise monotone maps of an interval, Acta Math. Univ. Comenian. (N.S.) 60 (1991), 3-10.

[5] K. Ciepliński, M. C. Zdun, On semi-conjugacy equation for homeomorphisms of the circle, in: Functional Equations-Results and Advances, Adv. Math. (Dordr.) 3, Kluwer Acad. Publ., Dordrecht, 2002, 135-158.

[6] K. Ciepliński, M. C. Zdun, On uniqueness of conjugacy of continuous and piecewise monotone functions, Fixed Point Theory Appl. 2009, Art. ID 230414, 11 pp.

[7] I. P. Cornfeld, S. V. Fomin, Ya. G. Sinař, Ergodic Theory, Grundlehren Math. Wiss. 245, Springer, New York, 1982. 
[8] N. A. Fotiades, M. A. Boudourides, Topological conjugacies of piecewise monotone interval maps, Int. J. Math. Math. Sci. 25 (2001), 119-127.

[9] C. Kawan, On expanding maps and topological conjugacy, J. Difference Equ. Appl. 13 (2007), 803-820.

[10] S. Kolyada, L'. Snoha, Some aspects of topological transitivity - a survey, in: Iteration Theory (Batschuns, 1992), Grazer Math. Ber. 334, Karl-Franzens-Univ. Graz, Graz, 1997, $3-35$.

[11] M. Kuczma, B. Choczewski, R. Ger, Iterative Functional Equations, Encyclopedia Math. Appl. 32, Cambridge Univ. Press, Cambridge, 1990.

[12] J. Matkowski, Integrable solutions of functional equations, Dissertationes Math. (Rozprawy Mat.) 127 (1975), 68 pp.

[13] W. de Melo, S. van Strien, One-dimensional Dynamics, Ergeb. Math. Grenzgeb. (3) 25, Springer, Berlin, 1993.

[14] J. Milnor, W. Thurston, On iterated maps of the interval, in: Dynamical Systems (College Park, MD, 1986-87), Lecture Notes in Math. 1342, Springer, Berlin, 1988, 465-563.

[15] Z. Nitecki, Differentiable Dynamics. An Introduction to the Orbit Structure of Diffeomorphisms, M.I.T. Press, Cambridge, Mass.-London, 1971.

[16] D.-S. Ou, K. J. Palmer, A constructive proof of the existence of a semi-conjugacy for a one dimensional map, Discrete Contin. Dyn. Syst. Ser. B 17 (2012), 977-992.

[17] Y.-G. Shi, Non-monotonic solutions and continuously differentiable solutions of conjugacy equations, Appl. Math. Comput. 215 (2009), 2399-2404.

[18] Y.-G. Shi, L. Li, Z. Leśniak, On conjugacy of r-modal interval maps with non-monotonicity height equal to 1, J. Difference Equ. Appl. 19 (2013), 573-584.

[19] M. C. Zdun, On conjugacy of multivalent functions on the circle, Rocznik Nauk.-Dydakt. Prace Mat. 17 (2000), 271-282.

[20] M. C. Zdun, On conjugacy of some systems of functions, Aequationes Math. 61 (2001), 239-254.

[21] M. C. Zdun, On conjugacy of homeomorphisms of the circle possessing periodic points, J. Math. Anal. Appl. 330 (2007), 51-65. 